\title{
Apoio ao planejamento do transporte metropolitano sustentável
}

\author{
Luiz Fernando de Melo Correia ${ }^{1}$, Maria Lucia Galves ${ }^{2}$ \\ ${ }^{1}$ Departamento de Geotécnica e Transportes - FEC-UNICAMP, Campinas, Brasil, luizfmc7@terra.com.br \\ ${ }^{2}$ Departamento de Geotécnica e Transportes - FEC-UNICAMP, Campinas, Brasil, mlgalves@fec.unicamp.br
}

\section{Recebido:}

15 de novembro de 2017

Aceito para publicação:

21 de agosto de 2018

Publicado:

30 de abril de 2019

Editor de área:

Cira Pitombo

\section{Palavras-chaves:}

Transporte metropolitano, Planejamento de transportes, Sustentabilidade,

Participação.

\section{Keywords:}

Metropolitan Transportation,

Transportation Planning,

Sustainability,

Participation.

DOI:10.14295/transportes.v27i1.1513

OPEN

ACCESS

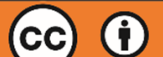

\begin{abstract}
RESUMO
Promover o transporte sustentável em regiões metropolitanas é um problema complexo, devido à grande quantidade de atores com objetivos muitas vezes conflitantes, além da forma com que cada comunidade, de acordo com o contexto local, absorve os conceitos ligados à sustentabilidade. Neste ambiente, é fundamental um suporte para orientar o planejamento e a tomada de decisão, considerando a sustentabilidade. A partir dos requisitos para um transporte sustentável e a participação dos diversos atores, elaborou-se um procedimento que auxilia na operacionalização do suporte, possibilitando a obtenção dos objetivos fundamentais dos atores, a proposição de alternativas e sua avaliação. Para demonstrar sua viabilidade, a proposta foi aplicada na Região Metropolitana da Baixada Santista, uma região importante localizada na Região Sudeste do Brasil. Trinta e nove objetivos fundamentais foram obtidos.
\end{abstract}

\begin{abstract}
Promoting sustainable transport in metropolitan regions is a complex problem, due to the large amount of actors with often conflicting goals, as well as the manner in which each community, in accordance with the local context, absorbs the concepts linked to sustainability. In this environment, it is vital a support to guide the planning and decision making, whereas sustainability. From the requirements for a sustainable transport and the participation of various actors, we elaborated a procedure that assists in the operationalization of support, enabling the achievement of the fundamental objectives of the actors, the proposition of alternatives and their evaluation. To demonstrate its viability, the proposal was applied in the Metropolitan Region of Baixada Santista, an important region located in the southeastern region of Brazil. Thirty-nine fundamental objectives were established.
\end{abstract}

\section{INTRODUCÃO}

O planejamento é um elemento crucial para qualquer iniciativa de desenvolvimento, incluindo a busca da sustentabilidade urbana (Rahman, 2016). Em áreas metropolitanas, a gravidade e complexidade dos problemas requerem abordagens e soluções inovadoras (Lalehpour, 2016). Sem uma gestão metropolitana adequada, a tentativa de resolução dos problemas pelos municípios, de forma individual, pode não ter o efeito esperado. Os resultados são políticas deficientes de transportes públicos, com elevadas taxas de utilização de veículos motorizados individuais (Ulian, 2008). Outra dificuldade é a coordenação entre o planejamento urbano e o dos transportes, geralmente complexa, devido às especificidades dos projetos de transporte regional e metropolitano. Muitas vezes, o planejamento de transportes enfatiza as regiões centrais das metrópoles, negligenciando a periferia (Liu e Alain, 2014). 
Segundo Sagaris (2014), pensar os cidadãos de uma metropóle como planejadores por direito abre caminho para estratégias mais eficazes para alcançar os objetivos da sustentabilidade nos transportes. Esta participação é fundamental, tendo em vista a resistência pública à mudança de comportamento, em busca de uma sociedade mais sustentável (Whitmarsh, 2012).

Neste contexto, os planejadores de transportes identificam a necessidade de maior coordenação e colaboração entre os atores, como etapas fundamentais para abordar questões relacionadas ao planejamento da rede de transportes e iniciativas de sustentabilidade (Beiler, 2016).

A Comissão Europeia para o Meio Ambiente (EEA, 2006) observa que o planejamento do transporte exige uma visão a longo prazo. As soluções precisam ser propostas com base em ampla consulta ao público e outras partes interessadas, e os objetivos devem refletir a situação local. Por outro lado, algumas abordagens para implementação de sistemas de transporte mais sustentáveis são tão ambiciosas que não fornecem qualquer orientação sobre como equilibrar os objetivos concorrentes e como os objetivos do sistema interagem (Goldman e Gorhan, 2006).

A dificuldade para obtenção desses objetivos é ainda maior em regiões metropolitanas, tendo em vista que existem características diferentes entre as regiões e os municípios de cada região, além de estratégias e políticas próprias para o planejamento dos transportes, incluindo a visão dos atores sobre a sustentabilidade (Rodrigues da Silva et al., 2008).

Alguns autores já desenvolveram metodologias que buscam agregar os objetivos conflitantes para o desenvolvimento de transportes sustentáveis, tais como: Yedla e Shrestha (2003), por meio da construção de cenários ou opções de sistemas de transportes para a cidade de Nova Deli, na Índia; Silva et al. (2008), pela definição das dimensões da mobilidade sustentável em regiões brasileiras, observando os objetivos dos atores; e Jones et al. (2013), utilizando a visão dos atores integrada em níveis diferentes dentro de várias hierarquias, considerando os conceitos gerais da sustentabilidade.

Todavia, existe a carência de uma metodologia que possa ser aplicada a qualquer região metropolitana, respeitando, porém, as características de cada contexto. Assim, este trabalho visa desenvolver um suporte para orientar o planejamento do transporte metropolitano considerando a sustentabilidade e desenvolver um procedimento para operacionalizá-lo.

Visando explicitar os conceitos necessários ao desenvolvimento da proposta, no próximo item serão analisadas as especificidades do transporte metropolitano sustentável. No item 3, será apresentada a metodologia, dividida em duas etapas: uma fundamentação teórica sobre o Auxílio Multicritério à Decisão, que servirá de suporte para a estruturação, avaliação e recomendação, e a descrição do procedimento proposto. Na sequência, será feita uma aplicação na Região Metropolitana da Baixada Santista. 0 item 5 contém as discussões sobre os resultados, seguidas das considerações finais.

\section{PLANEJAMENTO DE TRANSPORTES METROPOLITANOS SUSTENTÁVEIS}

\subsection{Transporte Metropolitano Sustentável}

A partir da priorização do desenvolvimento sustentável pelos países, que começou a emergir entre as décadas de 1980 e 1990, a sustentabilidade da infraestrutura tornou-se uma área de interesse nos campos da pesquisa, educação e aplicação prática (Jeon e Amekudzi, 2005). No 
setor de transportes, como resultado da aplicação dos conceitos de sustentabilidade, para atenuar as suas externalidades negativas, surgiu uma nova expressão denominada transporte sustentável (Qureshi, 2007).

Segundo Litman (2009), o termo transporte sustentável é uma continuação lógica do desenvolvimento sustentável, sendo utilizado para descrever os modos de transportes e sistemas de planejamento que são coerentes com as preocupações mais amplas da sustentabilidade. 0 transporte sustentável se contrapõe à visão pouco sustentável que requer grande energia para a construção e manutenção da infraestrutura, facilita a dispersão do uso do solo e utiliza grandes quantidades de combustíveis vindos de fontes não renováveis.

Muitas cidades do mundo procuram estabelecer sistemas de transporte urbano mais sustentáveis visando reduzir acidentes, congestionamentos, poluição do ar e ruído e melhorar as interações sociais, reduzindo as injustiças criadas pelos sistemas de transportes contemporâneos (Gössling, 2016).

Em regiões metropolitanas específicas, pesquisadores tais como Pospischil e Mailer (2014), Angel e Blei (2016) e Wang et al. (2017) estudaram as ações necessárias para promover o transporte metropolitano sustentável, tais como: utilização de transportes não motorizados, incentivo à utilização dos transportes públicos e controle do uso do solo.

No entanto, poucas organizações metropolitanas observam em seu planejamento o impacto dos transportes em relação aos aspectos ambientais, sociais e econômicos, essenciais em um sistema de transporte sustentável (Jeon, Amekudzi e Guensler, 2007).

Assim, um primeiro requisito para atingir um transporte sustentável, embasado em Shiftan, Kaplan e Hakkert (2003) e Litman (2012), refere-se aos objetivos da sustentabilidade, ou seja, objetivos ambientais, sociais e econômicos aplicados aos transportes sustentáveis:

- Ambientais: utilizar energia limpa, reduzir resíduos, poupar recursos energéticos, prevenir e mitigar mudanças climáticas, preservar áreas livres.

- Sociais: permitir igualdade de acesso, proporcionar melhoria da saúde da população, auxiliar o desenvolvimento econômico, proporcionar melhoria de qualidade de vida da população, preservar a herança cultural, ser seguro.

- Econômicos: ser financiável, ser indutor de desenvolvimento econômico, ser eficiente, necessitar de custos reduzidos para implantação e operação.

Para Nijkamp et al. (2007), em ambientes complexos como regiões metropolitanas, o processo de planejamento de transportes deve envolver, além de especialistas e políticos, a comunidade local. Desta forma, o segundo requisito para um transporte metropolitano sustentável se refere à participação dos atores, tendo em vista que as decisões sobre o tema têm impacto direto na comunidade, conforme descrito por Litman (2003) e Sagaris (2014).

0 terceiro requisito se refere às medidas, tanto de políticas públicas como de planejamento. Geurs e Van Wee (2000) e Steg e Gifford (2005) propõem uma série de medidas de políticas públicas para o desenvolvimento dos transportes sustentáveis - tecnológicas, comportamentais, de planejamento físico, gestão da oferta, espaciais e econômicas. Litman (2012) observa a importância do planejamento para o desenvolvimento de um transporte sustentável.

O planejamento de transportes sustentáveis implica a utilização dos diferentes modos de transportes no que cada um tem de melhor, reconhecendo o valor do transporte não motorizado, a importância do transporte público e a redução, mas não a eliminação, do uso do automóvel. Em relação ao transporte metropolitano, cujos modos também demandam escolhas 
ou decisões, as opções para o deslocamento podem variar entre o motorizado coletivo e individual, e não motorizado individual (Vasconcellos, 2012).

\subsection{Apoio ao Planejamento de Transportes Metropolitanos Sustentáveis}

Com base nos requisitos para um transporte sustentável e nos modos de transporte metropolitano, elaborou-se um fluxograma para apoio ao planejamento de transportes metropolitanos sustentáveis (Figura 1).

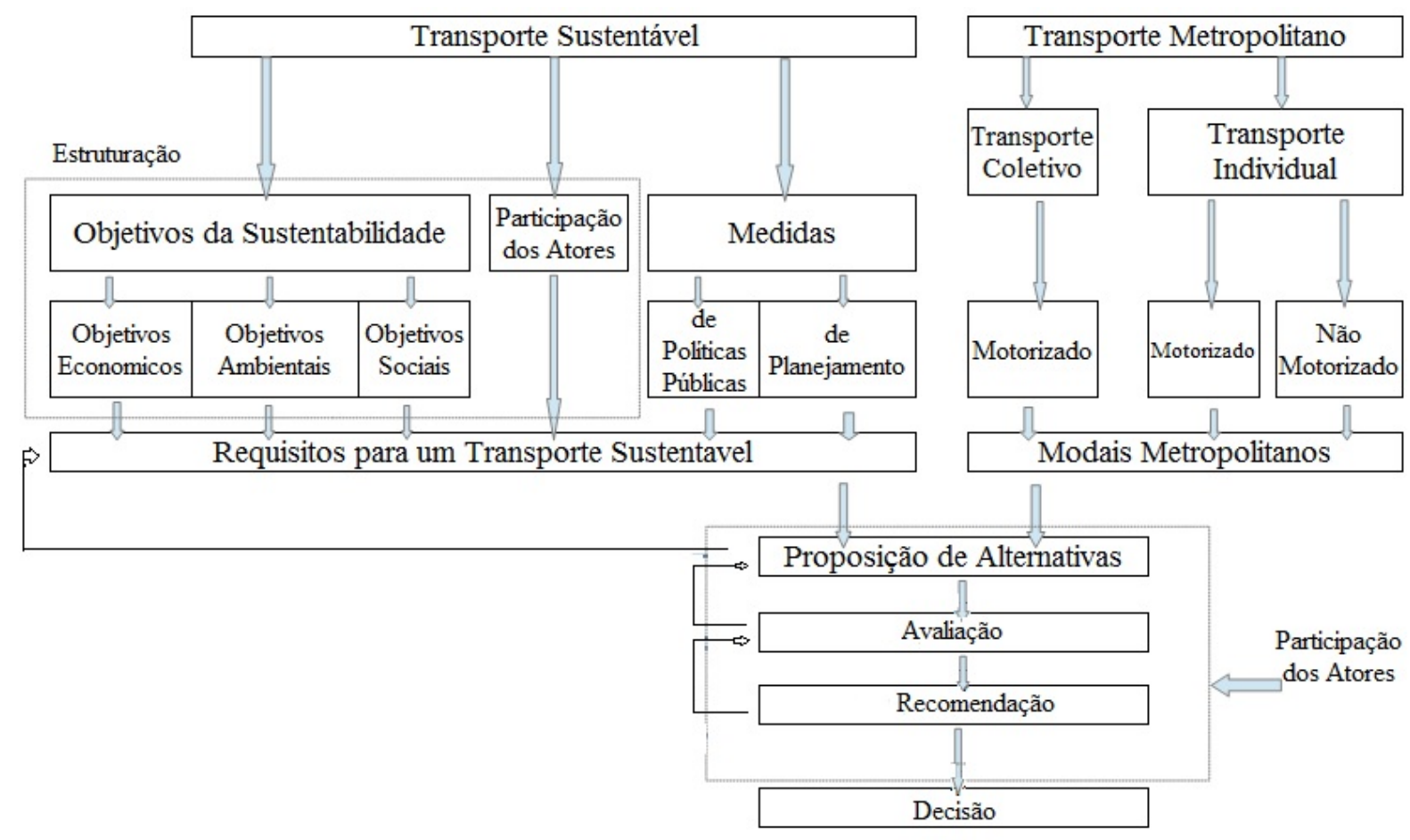

Figura 1. Fluxograma para apoio ao planejamento de transportes metropolitanos sustentáveis

O fluxograma foi organizado em dois pilares principais. Em um lado, o transporte sustentável, representado pelos objetivos da sustentabilidade, a participação dos atores e medidas de políticas públicas e de planejamento, que conduzem aos requisitos para um transporte metropolitano sustentável; de outro, os modos de transporte metropolitano, importantes para alcançar um sistema de transporte metropolitano sustentável.

Os requisitos para um transporte sustentável e os modos de transporte metropolitano são unidos para a proposição de alternativas, avaliação, recomendação e decisão.

O fluxograma é permeado pela participação dos atores, que, em um contexto metropolitano, possuem objetivos diversos. Conhecer os objetivos desses atores é essencial, já que a sustentabilidade é fortemente influenciada por características locais, conduzindo a elaboração de alternativas melhores. Isoladamente, os elementos do fluxograma ajudam a entender as necessidades de um sistema de transporte metropolitano sustentável, mas não auxiliam no processo de decisão. Justamente para integrar todos os elementos, conhecer os objetivos dos diversos atores, propor alternativas melhores e conduzir a uma decisão são utilizadas, como suporte, metodologias multicritério de auxílio à decisão. 
O Auxílio Multicritério à Decisão (AMCD), que tem como objetivo ajudar indivíduos ou grupos de indivíduos a explorar decisões importantes, pode ser subdividido em três fases: estruturação, avaliação e recomendação (Belton e Stewart, 2002). As três fases foram inseridas no fluxograma de acordo com cada elemento.

Assim, deve-se criar um procedimento para operacionalizar o suporte apresentado no fluxograma, orientando o planejamento e a tomada de decisão.

\section{METODOLOGIA}

Para o procedimento proposto são necessárias uma série de ações, conforme apresentado na Tabela 1.

Tabela 1: Procedimento para apoio ao planejamento de transportes metropolitanos sustentáveis

\begin{tabular}{lll}
\hline Item & Ações \\
\hline & 1 & Caracterização do contexto \\
& 2 & Identificação dos atores \\
& 3 & Escolha de um facilitador \\
& 4 & Obtenção dos objetivos \\
& 5 & Definição dos objetivos fundamentais \\
& 6 & Elaboração das hierarquias individuais \\
Estruturação & 7 & Validação das hierarquias individuais \\
& 8 & Elaboração da hierarquia conjunta \\
& 9 & Validação da hierarquia conjunta \\
& 10 & Validação final da hierarquia conjunta (reunião de conciliação) \\
& 11 & Pesquisa dos atributos \\
& 12 & Validação dos atributos e definição dos níveis de variação \\
& 13 & Proposição de alternativas \\
\hline Recomendação & 14 & Construção das funções de valor \\
& 15 & Constantes de escala \\
& 16 & Avaliação local das alternativas \\
& 17 & Avaliação global das alternativas \\
& 18 & Discussão dos resultados com os atores \\
\hline & &
\end{tabular}

Inicialmente, é importante caracterizar o contexto decisório, abrangendo características geográficas, populacionais e dos sistemas de transporte (Ação 1). Nesta etapa, também deve-se identificar os atores envolvidos com o transporte de passageiros na região em análise. Tais atores incluem os técnicos das prefeituras, das empresas de transporte - tanto privadas como estatais -, dos órgãos ou agências de controle regional de transportes, dos órgãos ambientais, das representações locais dos usuários de transportes motorizados ou não motorizados, bem como da população (Ação 2). 0 decisor pode ser um, ou mais de um, agente externo ou interno, governamental ou não, preocupado em promover o transporte metropolitano sustentável na região. É importante que o decisor (ou decisores) escolha(m) um facilitador, preferencialmente alguém que conheça os conceitos de transporte sustentável e tenha facilidade de comunicação com os diversos atores (Ação 3).

Uma vez definidos os atores e o facilitador, a etapa seguinte é a de entrevistas, que serão utilizadas, inicialmente, para obtenção dos objetivos fundamentais (Ações 4 e 5). 0 tipo de entrevista utilizado será não estruturado (Barros e Lehfeld, 2007). Para isso, deve ser efetuado um 
pequeno relato sobre o tema transporte sustentável, buscando-se os elementos relevantes para os diversos atores. Pretende-se utilizar uma entrevista focalizada, mas com aspectos de não dirigida. Em uma entrevista apenas focalizada poderiam ser restringidos alguns aspectos não abordados pelo entrevistador. Se fosse meramente não dirigida, o entrevistado poderia perderse em temas não coerentes com o problema em estudo.

Após a apresentação de um texto curto abrangendo os conceitos da sustentabilidade e as características de um transporte sustentável, são efetuadas três perguntas ao ator entrevistado:

1. Quais são os piores aspectos da movimentação de pessoas da Região Metropolitana?

2. Quais os melhores aspectos do transporte de passageiros da Região Metropolitana?

3. Observando os conceitos de sustentabilidade, quais os aspectos que poderiam ser implementados e quais as eventuais dificuldades?

As respostas formam uma lista de objetivos. Para cada objetivo da lista, pretende-se efetuar a seguinte pergunta aos atores: "Por que esse objetivo é importante no contexto de decisão?" Os objetivos importantes por si serão os objetivos fundamentais, e os demais serão os objetivosmeio; a seguir, são hierarquizados os objetivos fundamentais (Keeney, 1992). Recomenda-se organizar os objetivos fundamentais de acordo com os três grupos principais de objetivos da sustentabilidade: ambiental, social e econômico (Ação 6). Assim, no topo da hierarquia está a promoção do transporte metropolitano sustentável; no nível abaixo, os objetivos da sustentabilidade; e no último nível, os objetivos definidos para o contexto da região metropolitana em estudo.

As hierarquias individuais são apresentadas pelo facilitador e recebem sugestões ou a aprovação dos atores (Ação 7). A validação (aprovação) de forma individual é importante para uma nova reflexão dos atores e, caso necessário, para uma complementação de eventuais objetivos não abordados anteriormente. Em seguida, deve-se elaborar uma hierarquia conjunta, apresentada novamente para validação de forma individual (Ações 8 e 9).

Finalizada a construção da hierarquia conjunta e a validação de forma individual com os atores, é realizada uma reunião de conciliação (Ação 10). A reunião de conciliação possui dois objetivos principais: a validação da hierarquia conjunta (Item estruturação) e a definição das constantes de escala (Item avaliação). Nesta reunião, convidam-se todos os atores. 0 facilitador deve apresentar a hierarquia conjunta e provocar uma discussão entre eles na busca de um consenso. Um dos resultados é a hierarquia conjunta validada, que representa as especificidades da região metropolitana em análise.

Inicia-se o processo de definição dos atributos (Ação 11). Para a escolha dos atributos, poderão ser utilizados os indicadores de sustentabilidade, mais precisamente aqueles indicadores coerentes com um transporte sustentável mencionados na literatura, e/ou consulta a especialistas. Um atributo deve ter um nome, uma escala de medida e um intervalo de variação, compreendido entre o melhor nível viável e o pior nível aceitável. Os atributos e seus níveis de variação também precisam ser validados (aprovados) pelos atores (Ação 12).

Para a construção das alternativas, recomenda-se observar os objetivos fundamentais da hierarquia conjunta e as medidas de políticas públicas e de planejamento para um transporte sustentável (Ação 13). Nesta etapa é fundamental também observar a construção das hierarquias individuais dos diversos atores.

A avaliação tem início com a construção das funções de valor (Ação 14). As funções de valor podem ser construídas por meio dos métodos de pontuação direta ou bissecção (Ensslin et al., 
2001). No primeiro método, definem-se níveis para cada atributo ordenados preferencialmente. Ao melhor nível viável é associado o valor 100 e ao pior nível aceitável, o valor 0 . Em seguida, o ator é solicitado a expressar o valor dos demais níveis. No método da bissecção é necessário definir apenas o melhor e o pior nível de cada atributo, aos quais são associados os valores 100 e 0, respectivamente. Pede-se, então, que o ator indique um nível do atributo cujo valor esteja na metade dos valores extremos (o melhor e o pior). Repete-se o procedimento para os dois subintervalos, obtendo-se mais dois pontos da função de valor.

As constantes de escala são obtidas primeiro de forma individual, após a validação da hierarquia conjunta de forma individual (Ação 15). Na reunião de conciliação, são apresentadas as constantes de escala, servindo de parâmetro de discussão. Os valores finais são definidos por conciliação dos atores. As constantes de escala são estimadas pelo método swing weights (Ensslin et al., 2001). Considera-se, inicialmente, que todos os atributos estejam no pior nível e pedese que o ator escolha um atributo que gostaria de passar para o melhor nível. A esse salto correspondem 100 pontos. Em seguida, pergunta-se ao ator qual atributo ele passaria do pior para o melhor nível em segundo lugar e quanto vale esse salto. Repete-se o procedimento até que se definam os saltos de todos os atributos. As magnitudes dos saltos são medidas em relação ao primeiro. Por fim, é feita a normalização, obtendo-se as constantes de escala.

Para cada alternativa é definido o nível dentro da função de valor de cada atributo (Ação 16). Com todos os valores dos níveis dos atributos e as constantes de escala (k) é possível a avaliação pelo método de agregação aditiva. As alternativas podem ser, então, comparadas de acordo com os resultados parcial e global (Ação 17). Após a avaliação, é importante que seja efetuada uma apresentação final aos atores, para eventuais sugestões e recomendações.

Efetua-se a avaliação com métodos de agregação multicritério, permitindo avaliações local e global das alternativas. 0 método de agregação escolhido será a Função de Valor Multiatributo na Forma Aditiva, expressada pela seguinte relação:

$$
\mathrm{V}(\mathrm{A})=\mathrm{k}_{1} \cdot \mathrm{v}_{1}(\mathrm{~A})+\mathrm{k}_{2} \cdot \mathrm{v}_{2}(\mathrm{~A})+\mathrm{k}_{3} \cdot \mathrm{v}_{3}(\mathrm{~A})+\ldots \quad+\mathrm{k}_{\mathrm{n}} \cdot \mathrm{v}_{\mathrm{n}}(\mathrm{A})
$$

em que $\quad \mathrm{V}(\mathrm{A}) \quad$ é o valor global da alternativa $A$.

$\mathrm{V}_{1}(\mathrm{~A}), \mathrm{v}_{2}(\mathrm{~A}), \ldots, \mathrm{V}_{\mathrm{n}}(\mathrm{A})$ a são os valores das funções da alternativa A para os atributos $1,2, \mathrm{n}$.

$\mathrm{k}_{1}, \mathrm{k}_{2}, \ldots, \mathrm{k}_{\mathrm{n}} \quad$ parâmetros de calibração.

n é o número dos atributos.

Na etapa 18, recomendação, apresentam-se os resultados aos atores. Após essa etapa, poderão ser efetuadas recomendações para melhoria ou adaptação da metodologia proposta.

\section{APLICAÇÃO NA REGIÃO METROPOLITANA DA BAIXADA SANTISTA}

\subsection{Estruturação}

Seguindo o procedimento proposto, apresenta-se uma aplicação na Região Metropolitana da Baixada Santista (RMBS). A região foi escolhida tendo em vista a sua diversidade de modos de transportes metropolitanos, a presença de grandes áreas de preservação ambiental, a presença do maior porto da América Latina, uma elevada taxa de propriedade de veículos motorizados de uso individual (automóveis e motocicletas), que provoca uma série de externalidades negativas, elevado uso de bicicletas e a complexidade de suas interações com os modos motorizados, multiplicidade de atores em nível local e metropolitano. 
A região é formada por nove municípios com uma população de 1.781 .620 habitantes (Brasil, 2014). Segundo Zündt (2006), a RMBS foi a segunda região metropolitana do Estado de São Paulo e a pioneira tanto no âmbito da Constituição Federal de 1988, como da Constituição Estadual de São Paulo de 1989.

Segundo a Agência Metropolitana da Baixada Santista (AGEM, 2006), nos municípios da RMBS há grande movimentação de automóveis, ônibus, veículos de carga, bicicletas e pedestres, nos deslocamentos diários para trabalho, estudo, negócios, comércio e serviços. A intensa movimentação de veículos gera altos índices de acidentes de trânsito e congestionamento, principalmente na região central da RMBS. Outro aspecto relevante é a intensa quantidade de viagens pendulares a RMSP.

Os atores foram escolhidos em função do seu envolvimento com as questões dos transportes na Região Metropolitana da Baixada Santista. Observando os requisitos para sustentabilidade, além dos técnicos ligados aos transportes, foram identificados atores que representam os usuários e a população da região. Não foi identificado um decisor no contexto deste trabalho. Entre todos os atores listados, após várias tentativas, obteve-se apoio de todas as prefeituras, com exceção de uma delas. Os seguintes atores, em um total de vinte, foram identificados e numerados para controle:

- Prefeituras Municipais: (1) Santos, (2) Guarujá, (3) Bertioga, (4) Cubatão, (5) São Vicente, (6) Praia Grande, (7) Itanhaém, (8) Mongaguá, (9) Peruíbe.

- Governo Estadual: (10) Empresa Metropolitana de Transportes Urbanos (EMTU), (11) Desenvolvimento Rodoviário S.A. (DERSA), (12) Agência Metropolitana da Baixada Santista (AGEM-BS), (13) Companhia Ambiental do Estado de São Paulo (CETESB-BS).

- Governo Federal: (14) Instituto Brasileiro do Meio Ambiente e dos Recursos Naturais (IBAMA).

- Outros: (15) Associação Brasileira de Ciclistas (ABC), (16) Moradores de Santos Conselho de Comunidades de Bairro, (17) Usuários dos Transportes de Santos - Conselho de Comunidades de Bairro, (18) Operador Privado - Viação Piracicabana/Consórcio Mobilidade, (19) Moradores de Bertioga - Associação de Comissões e Conselhos Municipais de Bertioga, (20) Usuários dos Transportes de Bertioga - Associação de Comissões e Conselhos Municipais de Bertioga.

Neste estudo, o primeiro autor do trabalho, atuou como facilitador. Dos vinte atores identificados, dezenove participaram. Durante a entrevista, apresentou-se um texto curto sobre os conceitos de sustentabilidade e de transportes sustentáveis. Após esta pequena apresentação, foram feitas as perguntas apresentadas no item 3. Obteve-se, assim, uma lista de objetivos, que incluem objetivos-meio e objetivos fundamentais.

Aplicando o procedimento para definição dos objetivos fundamentais, foram explorados cada um dos objetivos listados na Ação 4, até encontrar os objetivos fundamentais de cada um dos atores. Nas entrevistas individuais, elaborou-se, também, a hierarquia de objetivos para cada ator. Para tanto, os objetivos fundamentais foram agrupados de acordo com os objetivos da sustentabilidade: ambientais, sociais e econômicos. Na mesma entrevista, a hierarquia foi apresentada ao ator que efetuou a sua validação ou solicitou alterações. Assim, ganhou-se tempo, tendo em vista que a agenda dos atores muitas vezes é repleta de atividades. 0 tempo de duração, da apresentação até a validação da hierarquia, foi, em média, de uma hora e meia. 
O facilitador, em escritório, analisou todas as hierarquias de objetivos fundamentais individuais, observando eventuais objetivos em comum, redundantes e complementares. 0 resultado final é uma hierarquia de objetivos fundamentais conjunta que necessita ser validada pelos atores. Nesta etapa, inicialmente, apresentou-se a hierarquia conjunta para os atores, em nova reunião, de forma individual, com todos os atores, explicando eventuais dúvidas e ouvindo eventuais sugestões ou efetuando simplesmente a validação da hierarquia conjunta.

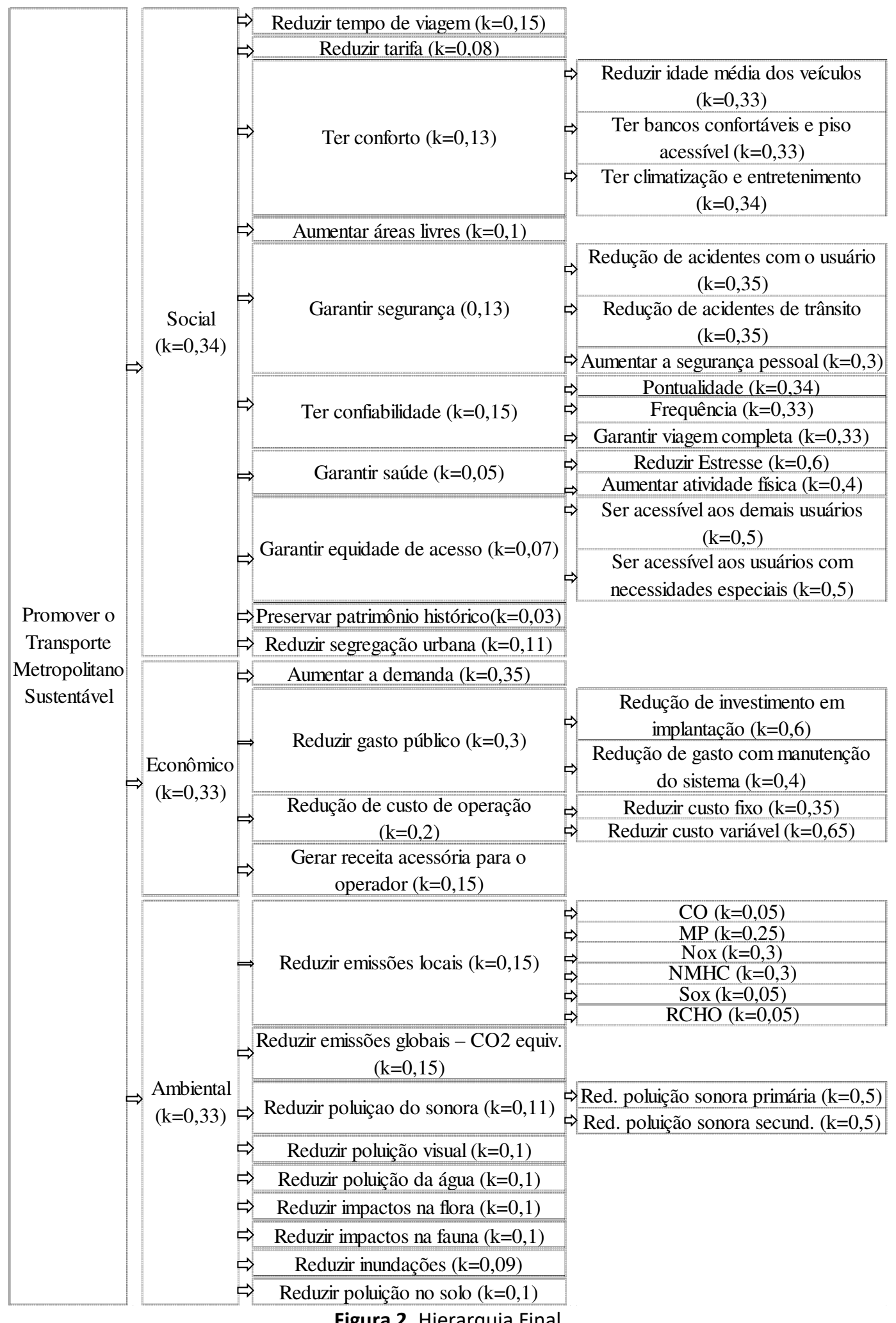

Figura 2. Hierarquia Final 
Para validação final foi realizada uma reunião de conciliação na sede da Agência Metropolitana da Baixada Santista, conduzida pelo facilitador. Todos os atores foram convidados, todavia, cinco compareceram. 0 resultado é a hierarquia apresentada na Figura 2.

Compareceram à Prefeitura de Santos, município núcleo da região; a Prefeitura de Praia Grande, a que mais cresce em termos populacionais; a Agência Metropolitana da Baixada Santista (AGEM), órgão do governo do Estado de São Paulo, que coordena a elaboração de planos metropolitanos; a Empresa Metropolitana de Transportes Urbanos (EMTU), responsável pelo planejamento e fiscalização do transporte metropolitano; e a Associação Brasileira de Ciclistas $(\mathrm{ABC})$, entidade não governamental que luta por avanços nos modos não motorizados. Durante as três horas de reunião foram apresentadas as hierarquias individuais dos diversos atores e a hierarquia final conjunta, como forma de promover um debate. Após uma pequena discussão por parte dos atores, efetuou-se a validação da hierarquia final.

Tabela 2: Atributos e Níveis de Variação

\begin{tabular}{|c|c|c|}
\hline Nome dos Atributos & Melhor Nível & Pior Nível \\
\hline Tempo Total de Viagem em relação ao Tempo Atual (\%) & -20 & 0 \\
\hline Tarifa Total em relação ao valor atual (\%) & -15 & 0 \\
\hline Idade Média dos Veículos (Anos) & 2 & 6 \\
\hline Climatização e Entretenimento (Tipo) & Ar,wi-fi,TV & Sem nada \\
\hline Características Internas (Bancose Piso) & \multicolumn{2}{|c|}{ Piso Rebaixado e Bancos Escada de Acesso e } \\
\hline Caracteristicas internas (Bancos e PISO) & Estofados & Bancos Rígidos \\
\hline Razão de Eficiência Espacial Estática (m²/passageiro) & 0,14 & 7,7 \\
\hline Acidentes de trânsito ( $\mathrm{N}^{\circ} / 10^{5}$ viagens) & 0 & 1 \\
\hline Acidentes com o usuário ( $\mathrm{N}^{\circ} / 10^{5}$ viagens) & 0 & 1 \\
\hline Assaltos aos veículos de transporte público ( $\mathrm{N}^{\circ} / 10^{5}$ viagens) & 0 & 20 \\
\hline Tempo máximo de espera do usuário no Horário de Pico (min) & 10 & 20 \\
\hline Índice de Cumprimento de Horários (atraso em min./\% total de viagens) & 1 & 5 \\
\hline Índice de Cumprimento de Viagens (\%) & 100 & 95 \\
\hline Índice de Estresse (\%) & 0 & $>66$ \\
\hline Tempo médio Diário por Atividade Física (min) & 70 & 0 \\
\hline Veículos, Terminais e Estações de Transporte Público com acessibilidade (\%) & 100 & $<100$ \\
\hline Tempo de acesso ao Transporte Público (min) & 2 & 20 \\
\hline Nível de Restrição a Intervenções de acordo com o Patrimônio (Nível de Restrição) & $\mathrm{P} 1 *$ & $\mathrm{P} 2 *$ \\
\hline Número de Passagens para Pedestres e Ciclistas ( $\left.\mathrm{n}^{\circ} / \mathrm{km}\right)$ & 10 & 4 \\
\hline Variação Média anual em relação a demanda atual (\%) & 3 & -2 \\
\hline 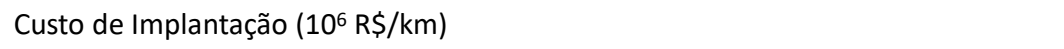 & $2 \times 10^{5}$ & $2 \times 10^{7}$ \\
\hline Custo de Manutenção Médio (R\$/km/Ano) & 3000 & 30000 \\
\hline Variação do custo fixo médio (\%) & 1 & 0 \\
\hline Custo Médio do Sistema por Passageiro (R\$/km/Ano) & 0,16 & 1,07 \\
\hline Receita Acessória sobre a Receita Operacional Média (\%) & 3 & 0 \\
\hline Variação de Óxido de Nitrogênio -Nox (\%) & -80 & 0 \\
\hline Variação de Material Particulado - MP (\%) & -50 & 0 \\
\hline Variação de Monóxido de Carbono - CO (\%) & -5 & 0,5 \\
\hline Variação de Hidrocarbonetos Não-Metano - NMHC (\%) & -20 & 0 \\
\hline Variação de Dióxido de Enxofre - SO2 (\%) & -50 & 0 \\
\hline Variação de Aldeídos - RCHO (\%) & -20 & 0 \\
\hline Variação de $\mathrm{CO}_{2}$ EQUI. (\%) & -43 & 0 \\
\hline Nível de Ruído Primário (dB (A)) & $<30$ & $>85$ \\
\hline Nível de Ruído Secundário (dB (A)) & $<30$ & $>45$ \\
\hline Descaracterização da Paisagem Local (Modos) & Enterrados & Elevados \\
\hline $\begin{array}{l}\text { Índice de Qualidade das Águas para Proteção da Vida Aquática e de Comunidades } \\
\text { Aquáticas (IVA) }\end{array}$ & $<2,5$ & $>=6,8$ \\
\hline
\end{tabular}


Tabela 2: Atributos e Níveis de Variação (continuação)

\begin{tabular}{lll}
\hline Nome dos Atributos & Melhor Nível & Pior Nível \\
\hline Supressão de vegetação (\%) & F1* & F2* \\
Grau de Extinção das espécies (Nível de extinção) & Fa1* Legenda) & Fa2* Legenda) \\
Variação da Impermeabilização dos solos (\%) & 0 & 10 \\
Quantidade de contaminante no solo (mg/kg) & VRQ & VI \\
\hline
\end{tabular}

P1 - Não existem jazidas arqueológicas, sítios arqueológicos. bens tombados e patrimônio imateriais registrados

P2 - Existência de jazidas, sítios arqueológicos e/ou inscrições rupestres sem a devida pesquisa, bens tombados e patrimônio imaterial registrado. Existência de bens em processo de tombamento e processo de registro de patrimônio imaterial

F1 - Qualquer \% de supressão em área agrícolas, florestas plantadas, ou desprovidas de vegetação nativa, fora de APP

F2 - Regiões de Mata Atlântica com Veg. Primária, Florestas em áreas rurais, qualquer necessidade de supressão em locais com espécies da flora nas listas de extinção, classificadas como "Extinta da Natureza", "Criticamente em Perigo", "Em Perigo" e "Vulnerável”

Fa1 - Existência de espécies com a classificação de "Menos Preocupante", “Dados Insuficientes", "Não Aplicável” e “Não Avaliada"

Fa2 - Existência de espécies classificadas como "Extinta da Natureza", "Criticamente em Perigo", "Em Perigo" e "Vulnerável”, sem possibilidade de manejo.

Observando os objetivos dos atores e as medidas de políticas públicas e de planejamento para um transporte sustentável, elaboraram-se duas alternativas, que foram apresentadas para alguns atores para discussão. A Alternativa 1, além de medidas de políticas públicas em transportes sustentáveis, possui maiores investimentos em sistemas de transportes, já em estudo para a região, incluindo sua infraestrutura. A Alternativa 2 possui menos investimentos em modos e sua infraestrutura, porém mais ações de medidas de políticas públicas. A situação atual e as alternativas elaboradas têm a seguinte descrição:

Situação Atual: Transporte hidroviário ligando o Centro de Santos ao Distrito de Vicente de Carvalho (Guarujá) e Centro de Bertioga ao extremo leste do Guarujá. VLT entre a Ponte dos Barreiros em São Vicente e Terminal Porto em Santos, integrando com ônibus intermunicipais. Ônibus intermunicipais convencionais. Dois terminais de integração, ônibus intermunicipais e municipais, em Praia Grande. Nenhum tipo de restrição de estacionamento. Nenhum programa de vistoria veicular para fins de emissão de gases. Horários dos itinerários dos ônibus intermunicipais, barcas e VLT disponíveis na Internet, mas sem controle em tempo real. Integração entre modos não motorizado e motorizado apenas nas barcas de passageiros (acesso a bicicletas). Existe malha cicloviária intermunicipal interligada entre Santos e São Vicente.

Alternativa 1: Expansão do hidroviário intermunicipal até Cubatão, Bertioga e Ligação de Vicente de Carvalho com a Ponta da Praia em Santos e Santa Rosa para o Centro de Santos. Ampliação do VLT até o Centro de Santos, Samarita e Variante Tude Bastos. Integração temporal e tarifária entre hidroviário, ônibus intermunicipais, VLT e ônibus municipais (Bilhete Único). Construção de terminal de integração em Bertioga entre os ônibus intermunicipais e municipais. Substituição da frota intermunicipal de ônibus por veículos híbridos. Integração das malhas cicloviárias municipais com os terminais intermunicipais. Implantação de programa de inspeção veicular obrigatória metropolitana. Criação de um centro de controle integrado dos modos intermunicipais de transportes, com rastreamento dos veículos. Restrição de vagas de estacionamento próximo aos corredores de transporte público e terminais intermunicipais, com exceção dos terminais extremos do VLT. Restrição de circulação de veículos em regiões de grande movimentação de pedestres.

Alternativa 2: Expansão do transporte hidroviário até Bertioga. Descentralização dos centros e atividades com a criação de um Plano Diretor e de Zoneamento da Região Metropolitana, para definição de corredores de elevada densidade. Restrição de vagas de estacionamentos públicos $e$ 
privados nos polos metropolitanos. Utilização de ferramentas de controle dos itinerários e horários dos modos motorizados pelos usuários (internet/aplicativo) em tempo real. Expansão da integração entre motorizados e não motorizados (bicicletas no VLT e ônibus). Integração tarifária e temporal entre municipais e intermunicipais e inter-intermunicipal (Bilhete Único). Subsídio ao transporte público e implantação de programa de controle de emissões veiculares metropolitanos. Criação de um Centro de Controle Integrado dos modos intermunicipais, com rastreamento dos veículos e possibilidade de ampliação da oferta em momentos específicos.

\subsection{Avaliação}

Desenvolveram-se as funções de valor com o apoio dos especialistas da EMTU, Prefeitura Municipal de Santos, Viação Piracicabana, CETESB, Secretária do Meio Ambiente do Estado de São Paulo e DERSA. Inicialmente, obteve-se as constantes de escala de todos os atores, de forma individual, na validação individual das hierarquias conjuntas. Tal procedimento é importante para que os atores tenham um parâmetro de comparação, na definição final, que deve ser efetuada durante a reunião de conciliação.

Na reunião de conciliação, os atores consideraram que os três objetivos principais da sustentabilidade possuem o mesmo peso. Para que a somatória dos pesos fosse igual a 1, conforme determinada a literatura, foi efetuado o arredondamento em uma fração, no peso dos objetivos sociais, escolhido em comum acordo entre os atores.

Para cada alternativa, foi definido o nível dentro da função de valor de cada atributo, e apresentados na Tabela 3. Em posse de todos os níveis dos atributos e das constantes de escala, efetuou-se o lançamento na expressão na forma aditiva, conforme item 3. 0 resultado global também é apresentado na Tabela 3. Níveis qualitativos são indicados por "N" e um valor numérico, conforme os níveis dos atributos apresentados na Tabela 2.

Tabela 3: Avaliação das Alternativas

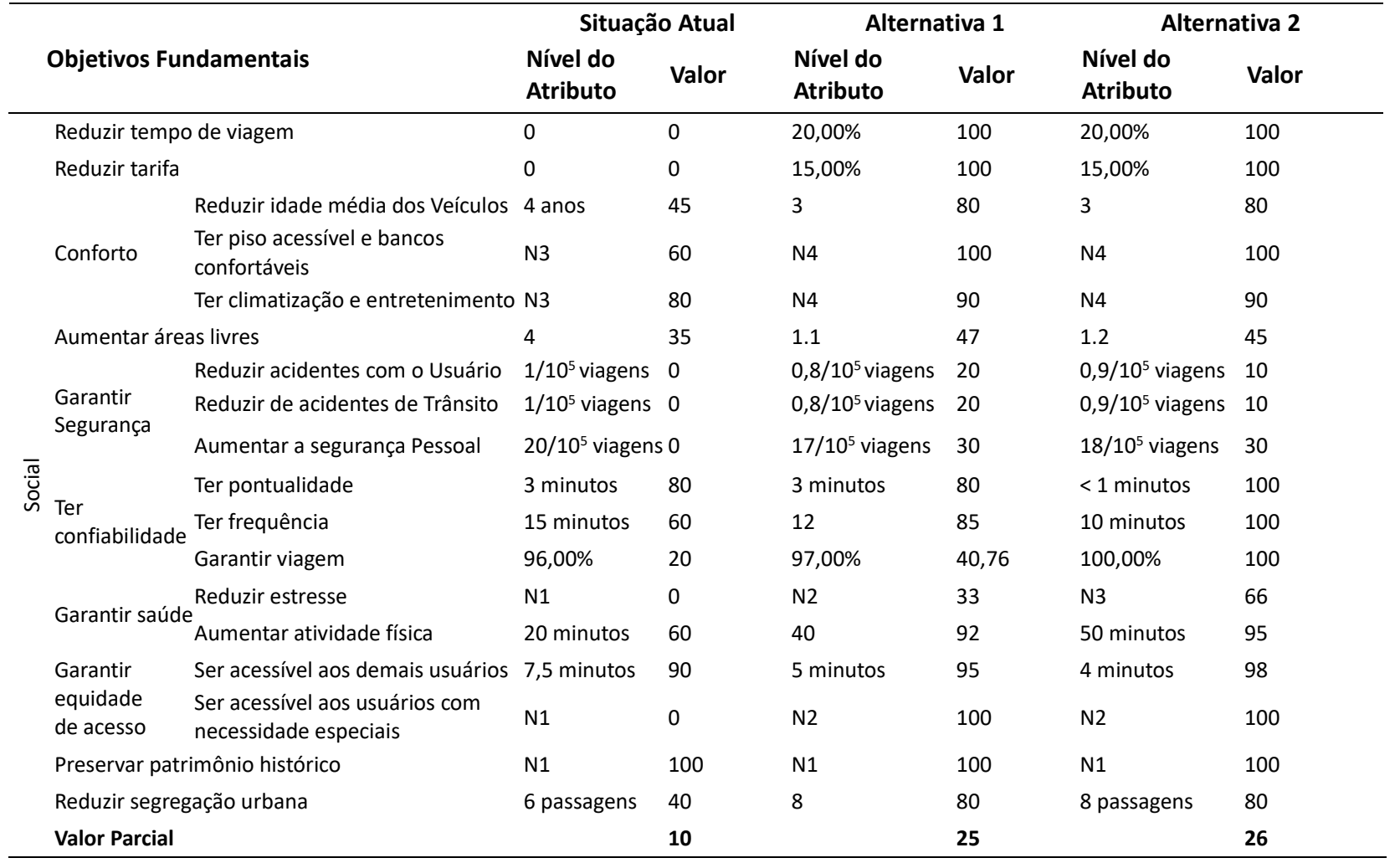


Tabela 3: Avaliação das Alternativas (continuação)

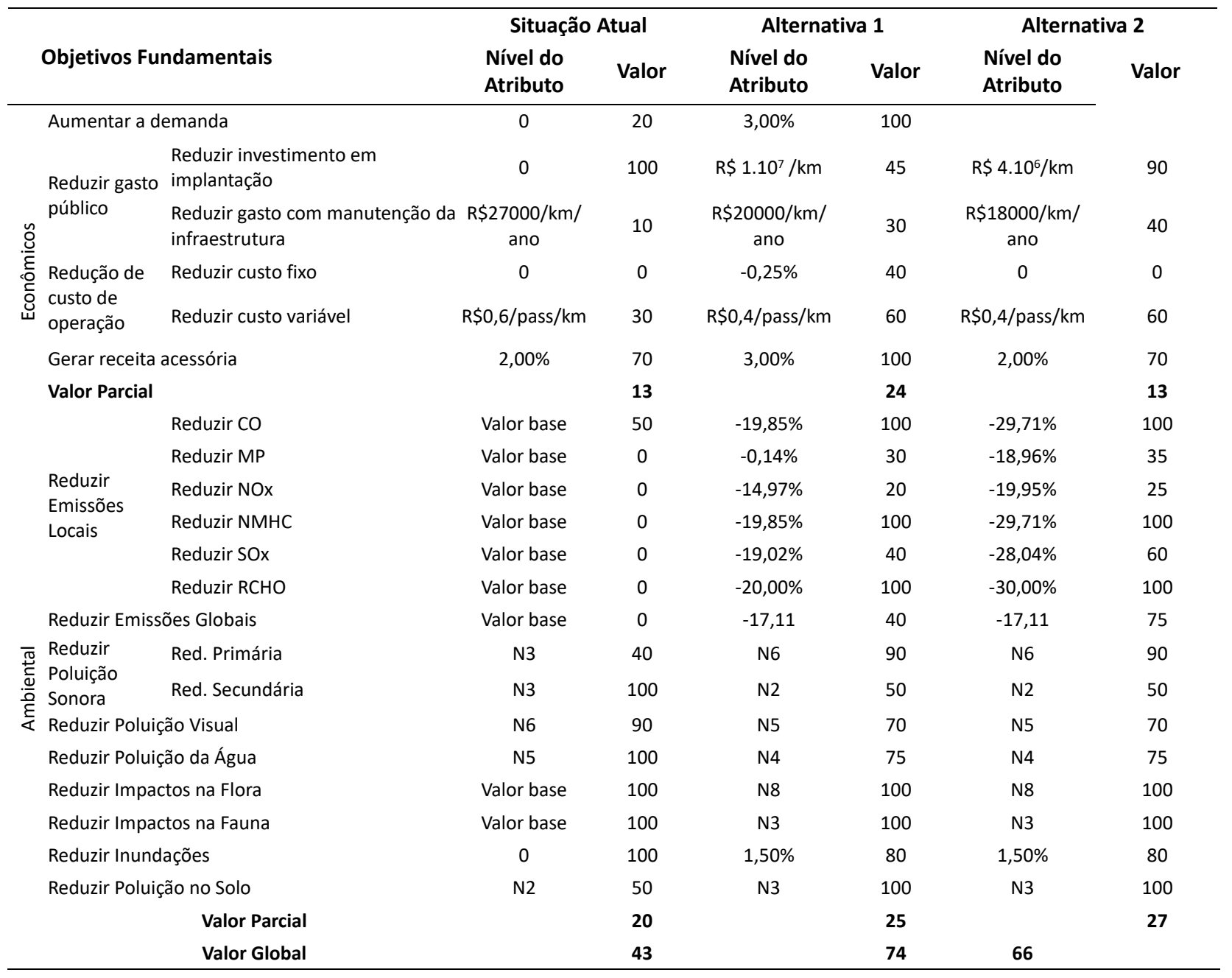

\subsection{Recomendação}

Após apresentação dos resultados os atores concordaram com os valores obtidos, sem questionamentos.

\section{DISCUSSÕES}

Inicialmente, é importante destacar que, dos 20 atores listados para a região, 19 participaram das entrevistas.

Durante as entrevistas é nítida a diferenciação de interesses entre os diversos atores. Por um lado, os atores mais "técnicos" possuem muitos objetivos sociais e econômicos, e alguns objetivos ambientais. Os moradores e usuários possuem quase unicamente objetivos sociais. Por outro lado, os técnicos dos órgãos ambientais não identificam apenas objetivos nesta área, demonstrando, também, terem muitos objetivos sociais.

Os objetivos fundamentais obtidos têm grande correlação com os objetivos da sustentabilidade, apresentados no item 2 deste trabalho, fato que revela a importância do setor para o bemestar da população. Cabe destacar que nem todos os atores entrevistados são técnicos experientes no setor de transportes de passageiros e nem mesmo possuíam algum tipo de conhecimento sobre os conceitos de transporte sustentável. 
Um dos resultados da aplicação do procedimento na Região Metropolitana da Baixada Santista foi uma hierarquia conjunta com 39 objetivos fundamentais, organizados entre ambientais, econômicos e sociais. Do total, 18 objetivos são sociais, 6 econômicos e 15 ambientais.

Nos objetivos sociais se observa que os atores têm muitas preocupações similares, como reduzir tempo de viagem, reduzir tarifa, ter conforto, garantir equidade de acesso, demonstrando estes serem pontos importantes em um planejamento de transporte metropolitano. Tal fato também foi observado na definição das constantes de escala, com exceção do objetivo reduzir tarifa, que obteve um peso menor, pois os atores consideram que, mesmo sendo um objetivo a ser atingido, o valor da tarifa no Brasil é pago, em parte, pelos empregadores.

Nos aspectos econômicos, as maiores preocupações estão na redução do gasto público e no aumento da demanda, o que demonstra, mais uma vez, o quanto são limitados os recursos para o setor e quanto é importante o planejamento de transportes. Esses dois objetivos obtiveram as maiores constantes de escala.

É importante destacar que a somatória das constantes de escala do objetivo fundamental "aumentar a demanda" e "reduzir gasto público" resulta em $65 \%$ do peso total dos objetivos econômicos. Assim, uma combinação de ações que levem a uma maior demanda pelos transportes públicos metropolitanos é essencial para manter a vitalidade do sistema.

Nos aspectos ambientais, após a validação da hierarquia conjunta, os atores optaram por manter constantes de escalas próximas, demonstrando que não apenas a emissão de gases é relevante em relação aos transportes metropolitanos, mas também objetivos como reduzir a poluição visual e sonora, a poluição do solo e da água, entre outros.

Em relação à avaliação das alternativas, a situação atual demonstra que o sistema de transporte metropolitano está longe da sustentabilidade, de acordo com objetivos sociais (10 pontos) e econômicos (13 pontos). Os principais problemas estão na redução do tempo de viagem, redução de tarifa, segurança e garantia de saúde, nos objetivos sociais; e no aumento da demanda e custo variável, nos objetivos econômicos. Tais aspectos podem estar relacionados com o predomínio do transporte motorizado individual na região, já que, entre suas externalidades, está o aumento dos congestionamentos, reduzindo a mobilidade e aumentando os tempos totais de viagens, a redução da demanda do transporte público e o gasto excessivo de combustíveis.

Os melhores aspectos da "Situação Atual" estão nos aspectos ambientais. Neste objetivo, o valor parcial da alternativa foi de 20 pontos, fato que pode ser explicado pela grande utilização do transporte não motorizado na região, que reduz as emissões locais e globais.

A Alternativa 1 possui maior equilíbrio entre os objetivos sociais, ambientais e econômicos. Nos objetivos econômicos, ela obteve melhor desempenho do que a Situação Atual e do que a Alternativa 2, tendo em vista o grande aumento de demanda de transporte público e a redução do custo fixo do sistema. Por outro lado, está alternativa é a que demanda maiores investimentos de implantação.

A Alternativa 2 possui bom desempenho nos objetivos sociais e é a melhor pelos objetivos ambientais. Em relação aos objetivos ambientais, 27 pontos contra 20 da Situação Atual e 25 da Alternativa 1. Tal fato pode estar relacionado à migração aos modos de transportes não motorizados, provocada pela descentralização das atividades. Pelos aspectos econômicos, tendo em vista a mesma previsão de descentralização, ela tão ruim como a Situação Atual (13 pontos), refletindo que um provável aumento da mobilidade local pode provocar o esvaziamento rápido dos modos de transportes metropolitanos. 
No contexto final, a Alternativa 1 obteve a melhor pontuação total, 74 pontos contra 66 pontos da Alternativa 2 e 43 pontos da Situação Atual. Tanto na Alternativa 1 como na Alternativa 2, existem ganhos em relação à Situação Atual, embora, como apresentado, a Alternativa 2, nos objetivos econômicos, seja tão ruim como a Situação Atual.

Por outro lado, melhorias na Alternativa 2 não podem ser descartadas. Um dessas melhorias é um maior controle da provável "migração" do transporte metropolitano para o não motorizado, tendo em vista a descentralização das atividades. Essa "migração" precisa ocorrer de forma gradual, para que os operadores de transportes, tanto públicos como privados, possam adaptar sua operação ao novo cenário. Alterações nos contratos de concessão podem amenizar as perdas dos operadores. Além disso, a Alternativa 2 é a que demanda os menores investimentos, fato importante em uma era de recursos escassos para o setor. Pensando em investimentos, uma melhor programação das ações de infraestrutura da Alternativa 1 pode torná-la mais atraente pelos objetivos econômicos.

\section{CONSIDERAÇÕES FINAIS}

Este trabalho propôs um procedimento para apoio ao planejamento do transporte sustentável, respeitando o contexto de uma região metropolitana, permeado pela interação com todos os atores envolvidos com os transportes, incluindo a população, visando conhecer os valores fundamentais para a região em estudo e agregando a visão dos atores frente às questões do transporte sustentável.

A lista geral de objetivos auxilia os gestores de transportes metropolitanos a entender as virtudes e carências do sistema existente em relação à sustentabilidade, servindo de aprendizado para os atores e também para o facilitador, tendo em vista que, muitas vezes, tais questões não são discutidas e analisadas em profundidade na busca da essência dos problemas do setor. Mesmo havendo atores com pouca experiência no setor e, além disso, com pouco conhecimento sobre sustentabilidade aplicada ao setor de transportes, a lista de objetivos fundamentais gerada é abrangente e coerente com a literatura sobre o tema.

As alternativas não demandam apenas investimentos em novos modos de transportes, mas também melhorias de gestão, utilização de tecnologia, informação ao usuário, controle de emissões, campanhas educativas, entre outras ações. 0 ideal é uma composição de medidas que envolvam tecnologia, medidas governamentais, planejamento físico, mudanças de comportamento e qualidade de vida. Sem a interação da sociedade durante todo o processo, os resultados não podem ser consistentes com o esperado.

Sugerimos a aplicação da mesma metodologia em outras regiões metropolitanas do Brasil e do mundo, não apenas para verificar sua aplicabilidade, mas também para comparar os objetivos fundamentais, atributos, níveis de variação, constantes de escala, alternativas e avaliação. Mesmo que os resultados sejam similares, o caminho para encontrá-los permitirá aos planejadores de transporte metropolitano uma reflexão sobre a busca por um transporte mais sustentável.

\section{REFERÊNCIAS}

AGEM (2006) Estudos técnicos, levantamentos, diagnóstico e diretrizes para elaboração do plano cicloviário metropolitano da Região Metropolitana da Baixada Santista - PCM - BS. Disponível em: <http://www.agem.sp.gov.br/midia/PCM-SUMARIOAPRESENTACAO-E-INTRODUCAO-DO-PCM.pdf> (Acesso em: 05/10/2017).

Angel, S. e A. M. Blei (2016) The productivity of American cities: How densification, relocation, and greater mobility sustain the productive advantage of larger U.S. Metropolitan labor markets. Cities, n. 51, pp. 36-51. DOI: 10.1016/j.cities.2015.11.030. 
Barros, A. J. S. e N. A. S. Lehfeld (2007) Fundamentos da metodologia científica. São Paulo: Prentice Hall.

Beiler, M. O. (2016) Organizational sustainability in transportation planning: Evaluation of multi-jurisdictional agency collaboration. Journal of Transport Geography, n. 52, pp. 29-37. DOI: 10.1016/j.jtrangeo.2016.02.013.

Belton, V. e T. J. Stewart (2002) Multiple criteria decision analysis: an integrated approach. Norwell: Kluwer Academic Publishers. DOI: 10.1007/978-1-4615-1495-4.

Brasil (2014) Resolução no 2, de 26 de Agosto de 2017. População residente segundo as unidades da Federação. Ministério do Planejamento, Orçamento e Gestão. Diário Oficial da União, p. 98, 28 de Agosto. Disponível em: <https://www.jusbrasil.com.br/diarios/75661110/dou-secao-1-28-08-2014-pg-98> (Acesso em: 05/10/2016).

EEA (2006) Urban sprawl in Europe - The ignored challenge. Copenhagen: EEA. Disponível em: <https://www.eea.europa.eu/publications/eea_report_2006_10> (Acesso em: 05/10/2017).

Ensslin, L.; G. Montibeller Neto e S. M. Noronha, S. M. (2001) Apoio à decisão - Metodologia para estruturação de problemas e avaliação multicritério de alternativas. Florianópolis: Insular.

Geurs, K. e B. Van Wee (2000) Backcasting as a tool to develop a sustainable transport scenario assuming emission reductions of $80-90 \%$. Innovation, v. 13, n. 1, pp. 47-62. DOI: 10.1080/135116100111658.

Goldman, T. e R. Gorham (2006) Sustainable urban transport: four innovative directions. Technology in Society, n. 28, pp. 261273. DOI: $10.1016 /$ j.techsoc.2005.10.007.

Gössling, S. (2016) Urban transport justice. Journal of Transport Geography, v. 54, May, pp. 1-9. DOI: 10.1016/j.jtrangeo.2016.05.002.

Jeon, C. M. e A. Amekudzi (2005) Addressing sustainability in transportation systems: definitions, indicators, and metrics. Journal of Infrastructure Systems, March, pp.31-50. DOI: 10.1061/(ASCE)1076-0342(2005)11:1(31).

Jeon, C. M.; A. A. Amekudzi e R. L. Guensler (2007) Evaluating plan alternatives for transportation system sustainability: Atlanta Metropolitan Region. International Journal of Sustainable Transportation, v. 4, n. 4, pp. 227-247. DOI: $10.1080 / 15568310902940209$.

Jones, S.; M. Tefe e S. Appiah-Opoku (2013) Proposed framework for sustainability screening of urban transport projects in developing countries: a case study of Accra, Ghana. Transportation Research, Part A, v. 49, pp. 21-34. DOI: 10.1016/j.tra.2013.01.003.

Keeney, R. L. (1992) Value focused thinking: a path to creative decision making. Cambridge: Harvard University Press.

Lalehpour, M. (2016) Recognition of management structure and spatial planning in Tehran metropolitan area. Journal of Urban Management, v. 5, pp. 3-15. DOI: 10.1016/j.jum.2016.05.001.

Liu, L. e L. H. Alain (2014) Transport and land use interaction: a French case of suburban development in the Lille Metropolitan Area (LMA). Transportation Research Procedia, v. 4, pp. 120-139. DOI: 10.1016/j.trpro.2014.11.011.

Litman, T. (2003) Reinventing transportation - Exploring the paradigm shift needed to reconcile transportation and sustainability objectives. Canada: Victoria Transport Policy Institute. Disponível em: <https://pdfs.semanticscholar.org/e639/18634cd4777fe0c200192a0f839837a209a2.pdf> (Acesso em: 05/10/2017).

Litman, T. (2009) Sustainable Transportation and TDM.Online TDM Encyclopedia.Canada: Victoria Transport Policy Institute. Disponível em: <https://www.vtpi.org/tdm/tdm67.htm> (Acesso em: 10/03/2015).

Litman, T. (2012) Well measured: developing indicators for sustainable and livable transport planning. Canada: Victoria Transport Policy Institute. Disponível em: <http://www.vtpi.org/wellmeas.pdf> (Acesso em: 1/2/2015).

Nijkamp, P.; M. T. Borzacciello; B. Ciuffo e F. Torrieri (2007) Sustainable urban land use and transportation planning: a cognitive decision support system for the Napels Metropolitan Area. International Journal of Sustainable Transportation, v.1, pp.91-114. DOI: 10.1080/15568310601091981.

Pospischil, F. e M. Mailer (2014) The potential of cycling for sustainable mobility in metropolitan regions - the facts behind the success story of Innsbruck. Transportation Research Procedia, v. 4, pp. 80-89. DOI: 10.1016/j.trpro.2014.11.007.

Qureshi, I. A. (2007) Urban transport and sustainable transport strategies: a case study of Karachi, Pakistan. Tsinghua Science and Technology, June, v. 12, n. 3, pp. 309-317. ISSN: 1007-0214. DOI: 10.1016/S1007-0214(07)70046-9.

Rahman, A. U. (2016) Urban sustainability through strategic planning: a case of metropolitan planning in Khulna city, Bangladesh. Journal of Urban Management, v. 5, pp. 16-22. DOI: 10.1016/j.jum.2016.06.001.

Sagaris, L. (2014) Citizen participation for sustainable transport: the case of "Living City" in Santiago, Chile (1997-2012). Journal of Transport Geography, Dec., v. 41, pp. 74-83. DOI: 10.1016/j.jtrangeo.2014.08.011.

Steg, L. e R. Gifford (2005) Sustainable transportation and quality of life. Journal of Transport Geography, v. 13, pp. 59-69. D0I: 10.1016/j.jtrangeo.2004.11.003.

Silva, A. N. R.; M. S. Costa e M. H. Macedo (2008) Multiple views of sustainable urban mobility: the case of Brazil. Transport Policy, v. 15, n. 6, Special Issue, p. 350-360. DOI: 10.1016/j.tranpol.2008.12.003.

Ulian, F. (2008) Sistemas de transportes terrestres de passageiros em tempos de reestruturação produtiva na Região Metropolitana de São Paulo. Tese (Doutorado). São Paulo: Universidade de São Paulo, 2008.

Vasconcellos, E. A. (2012) Mobilidade urbana e cidadania. Rio de Janeiro: SENAC Nacional, 2012.

Wang, Y.; S. Mishra; X. Ye; L. Li e B. Wu (2017) The application of integrated multimodal metropolitan transportation model in urban redevelopment for developing countries. Transportation Research Procedia, v. 25, pp. 2990-3002. DOI: 10.1016/j.trpro.2017.05.378.

Whitmarsh, L. (2012) How useful is the multi-level perspective for transport and sustainability research? Journal of Transport Geography, v. 24, pp. 483-487. DOI: 10.1016/j.trpro.2017.05.378. 
Yedla, S.; Shrestha, R. M. (2003) Multi-criteria approach for the selection of alternative options for environmentally sustainable transport system in Delhi. Transportation Research Part A, v. 37, pp. 717-729. DOI: 10.1016/S0965-8564(03)00027-2.

Zündt, C. (2006) Baixada Santista: uso, expansão e ocupação do solo, estruturação de rede urbana regional e metropolização. Disponível em:

<http://www.nepo.unicamp.br/publicacoes/livros/vulnerabilidade/arquuivos/arquuivos/vulnerab_cap_11_pgs_305_336 .pdf> (Acesso em: 05/10/2017). 